\title{
Synthesis and Mesomorphic Properties of New Achiral Liquid Crystals with 3-Alkoxy-2-(alkoxymethyl)-1-propoxy Swallow-Tails ${ }^{\dagger}$
}

\author{
Kyung-Tae Kang," Jeong Tak Kim, Ryeo Yun Hwang, Song Ju Park, Seng Kue Lee, Jong Gun Lee, and Yong Bae Kim \\ Department of Chemistry and Chemistry Institute for Funtional Materials, Pusan National University, Pusan 609-735, Korea \\ ${ }^{*}$ E-mail: hytkang@pusan.ackr. \\ "Liquid Crystal Research Center and Department of Chemistry, Konkuk University, Seon 143-710, Korea \\ Received June 26, 2007
}

\begin{abstract}
New liquid crystalline (biphenylcarbonyloxy)benzoates with an achiral swallow-tail derived from 3-alkoxy-2(alkoxymethyl)-1-propanol [( $\left.\mathrm{ROCII})_{2} \mathrm{CIICH}_{2} \mathrm{OII}, \mathrm{R}=\mathrm{Me}, \mathrm{Et}, \mathrm{Pr}, \mathrm{Bu}\right]$ were prepared. These liquid crystals exhibited the phase sequence (I- $\$ \mathrm{~m} \Lambda$-SmCalt-( $\mathrm{SmCX})$-Cr) and showed antiferroelectric-like Smectic $\mathrm{C}$ phase (SmCalt) at temperature lower, and temperature range broader than do the compounds containing a branched alkyl group as a swallow-tail. The temperature ranges of antiferroelectric phase were found to be $30-90^{\circ} \mathrm{C}$ and crystallization temperatures were $4-60^{\circ} \mathrm{C}$. The binary mixture of an achiral swallow-tailed liquid crystal and a chiral antiferroelectric liquid crystal, (S)-MIPOBC showed antiferroelectric smectic $\mathrm{C}$ phase at temperature much lower than the single chiral antiferroelectric liquid crystal does.
\end{abstract}

Key Words : Liquid crystal, Swallow-tailed liquid crystal, Antiferroelectric-like phase, 3-Alkoxy-2-(alkoxymethyl)-1-propanol

\section{Introduction}

Achiral materials with terminal swallow-tailed moieties have been demonstrated to display 'antiferroelectric-like' phase, so-called SmCalt phase, and can be used as host components of antiferroelectric mixture.' Chiral swallowtailed materials derived from various types of chiral compounds were prepared and their mesomorphic properties were investigated. ${ }^{2}$ However, the variation of swallow-tailed moieties has been limited to branched alkyl groups due to the synthetic problems. The investigation of chemical structure and physical property relationship is very important to liquid crystal chemistry. To meet the demand and to obtain various liquid crystalline compounds of varying physical properties a new synthetic route has to be developed.

Recently we reported the synthesis of diverse achiral swallow-tailed compounds derived from 1,3-dialkoxy-2propanol $\left[\left(\mathrm{ROCH}_{2}\right)_{2} \mathrm{CHOH}\right]$ where $\mathrm{R}$ is methyl, ethyl, propyl, butyl, $\mathrm{CH}_{2} \mathrm{CF}_{3}{ }^{3}$ Herein we report the synthesis of new achiral swallow-tailed compounds derived from 3alkoxy-2-(alkoxymethyl-1-propanol and investigation of their mesomorphic properties.

\section{Experimental Section}

${ }^{1} \mathrm{H}-\mathrm{NMR}$ spectra were recorded on Varian Gemini-200 $(200 \mathrm{MHz})$ and Varian Inova $(500 \mathrm{MHz})$ spectrometer using chloroform as an internal standard. The latter instrument was also used for recording ${ }^{13} \mathrm{C} \mathrm{NMR}$ spectra in $\mathrm{CDCl}_{3}$ (solvent and internal reference). Elemental analyses were performed at the National Center for Inter-University Research

${ }^{\dagger}$ This paper is dedicated to Professor Sang Chul Shim on the occasion of his honorable retirement.
Facilities, Seoul National University. Phase transition temperature and phase appearance of final products were measured using polarizing microscope (Olympus BH-2) with a hot stage and a controller (Mettler FP-800-HT heating stage). Transition temperature and enthalpy were determined by differential scanning calorimetry (DSC) using a PerkinElmer DSC-7 calorimeter. Debenzylation of benzyl ethers to the corresponding alcohols and phenols were carried out in the Parr hydrogenation reactor (Parr 3916EKX).

Preparation of 2,2-dimethyl-5-(hydroxymethyl)-1,3dioxane 3 . The compounds 2 and 3 were prepared according to the literature method. ${ }^{4}$

Into the THF $(20 \mathrm{~mL})$ solution of triethyl methanetricarboxylate $(13.4 \mathrm{~g}, 57.7 \mathrm{mmol})$ was added borane-dimethyl sulfide complex in THF $(5 \mathrm{M}, 36 \mathrm{~mL}, 180 \mathrm{mmol})$ under argon atmosphere. The solution was heated under reflux for $8 \mathrm{~h}$ with distillation of dimethyl sulfide and THF. To the cooled solution was added methanol $(60 \mathrm{~mL})$ and stirred for $4 \mathrm{~h}$. The solvent was removed. Addition of methanol and evaporation of volatiles were repeated $(3 \times 30 \mathrm{~mL})$. The residue was purified by column chromatography (silica gel, chloroforms/ethanols 3:1, $\mathrm{R}_{\mathrm{f}}=0.25$ ) to afford 2-(hydroxymethyl)propane-1,3-diol (2, 6.01 g, 98\%). 'H NMR (DMSO$\left.\mathrm{d}_{6}\right) \delta 1.57$ (sept, $\left.1 \mathrm{H}, J=5.9 \mathrm{~Hz}\right), 3.37(\mathrm{dd}, 6 \mathrm{H}, J=5.9,5.1$ $\mathrm{Hz}), 4.28(\mathrm{t}, 3 \mathrm{H}, J=5.1 \mathrm{~Hz})$.

To a THF $(130 \mathrm{~mL})$ solution of 2-(hydroxymethyl)propane-1,3-diol ( $2,4.05 \mathrm{~g}, 38.2 \mathrm{mmol})$ containing 4-toluenesulfonic acid monohydrate $(0.28 \mathrm{~g}, 1.45 \mathrm{mmol})$ was added 2,2-dimethoxypropane $(6.6 \mathrm{~mL}, 5.59 \mathrm{~g}, 53.7 \mathrm{mmol})$. The solution was stirred for $3 \mathrm{~h}$ at room temperature and was neutralized by adding of triethylamine $(2.6 \mathrm{~mL}, 1.89 \mathrm{~g}, 18.6$ $\mathrm{mmol}$ ). The solvent was removed and the residue was chromatographed on silica gel (chloroform/ethanol 10:1, $\mathrm{R}_{\mathrm{f}}$ $=0.54)$ to give $3(5.17 \mathrm{~g}, 93 \%)$. 'H NMR $\delta 1.40(\mathrm{~s}, 3 \mathrm{H}), 1.45$ 
(s, 3H), 1.81-1.87 (m, 2H), 3.70-3.82 (m, 4H), 4.03 (dd, $2 \mathrm{H}$, $J=12.1,4.0 \mathrm{~Hz}$ ).

Preparation of 2,2-dimethyl-5-(benzyloxymethyl)-1,3dioxane 4. Sodium hydride ( $60 \%$ in mineral oil, $0.42 \mathrm{~g}, 10.6$ mmol) was added to a dry, two-necked round-bottomed flask equipped with a rubber septum, and was washed with THF $(2 \times 10 \mathrm{~mL})$. THF was removed to give $\mathrm{NaH}$ as a white powder. The alcohol $3(1.03 \mathrm{~g}, 7.05 \mathrm{mmol})$ in DMF was added to the flask. After an hour of stirring at room temperature, benzyl bromide $(2.40 \mathrm{~g}, 14.1 \mathrm{mmol})$ was added. The reaction mixture was refluxed for $12 \mathrm{~h}$. The reaction was quenched with water and the solution was extracted with diethyl ether. The combined organic layer was dried over $\mathrm{Na}_{2} \mathrm{SO}_{4}$, filtered, and concentrated. The residue was chromatographed on silica gel (hexanes/ether 1:1, $\mathrm{R}_{\mathrm{f}}=0.50$ ) to afford $4(1.42 \mathrm{~g}, 86.0 \%)$. 'H NMR $\delta 1.37(\mathrm{~s}, 3 \mathrm{H}), 1,41(\mathrm{~s}, 3 \mathrm{H})$, $2.16-2.29(\mathrm{~m}, 1 \mathrm{H}), 3.72(\mathrm{dd}, 2 \mathrm{H}, J=11.9,4.6 \mathrm{~Hz}), 3.99(\mathrm{dd}$, $2 \mathrm{H}, J=11.9,4.6 \mathrm{~Hz}), 4.25(\mathrm{~d}, 2 \mathrm{H}, J=7.3 \mathrm{~Hz}), 5.14(\mathrm{~s}, 2 \mathrm{H})$, 7.29-7.38 (m, 5H).

Preparation of 2-(benzyloxymethyl-propane)-1,3-diol 5. To an ethanol ( $3 \mathrm{~mL})$ solution of the benzyl ether $4(2.36$ $\mathrm{g}, 10.0 \mathrm{mmol}$ ) was added $5 \mathrm{~mL}$ of $3 \mathrm{~N}$ aqueous $\mathrm{HCl}$ and the mixture stirred at room temperature for $1 \mathrm{hr}$. The solvent ethanol was evaporated and the residue was extracted with ether. Purification by column chromatography (silica gel, chloroform/ethanol 10:1, $R_{i}=0.55$ ) gave 2-(benzyloxy methyl)-propane-1,3-diol $(5,1.94 \mathrm{~g}, 99.0 \%) .{ }^{\mathrm{H}} \mathrm{H}$ NMR $\delta$ 2.03 (sept, $1 \mathrm{H}, J=5.5 \mathrm{~Hz}$ ), 2.82 (br s, $2 \mathrm{H}$ ), 3.60 (d, $2 \mathrm{H}, J=$ $5.5 \mathrm{~Hz}), 3.80(\mathrm{~d}, 4 \mathrm{H}, J=5.5 \mathrm{~Hz}), 4.50(\mathrm{~s}, 2 \mathrm{H}), 7.25-7.35(\mathrm{~m}$, $5 \mathrm{H})$.

Preparation of 1,3-dialkoxy-2-(benzyloxymethyl)propanes 6. The synthesis of $6 a$ is typical. To a suspension of sodium hydride ( $60 \%$ in mineral oil, $0.39 \mathrm{~g}, 9.75 \mathrm{mmol}$ ) in THF $(5 \mathrm{~mL})$ was added a THF $(3 \mathrm{~mL})$ solution of the diol 5 $(0.64 \mathrm{~g}, 3.25 \mathrm{mmol})$ and the mixture stirred for $1 \mathrm{~h}$ at room temperature. Methyl iodide $(0.28 \mathrm{~g}, 19.5 \mathrm{mmol})$ was added to the resulting solution and the mixture was refluxed for 24 $h$. The reaction was quenched with water and the crude product was extracted with ether. The combined organic layers were dried over $\mathrm{Na}_{2} \mathrm{SO}_{4}$, filtered, and concentrated. The residue was chromatographed on silica gel (hexanes/ ether $\left.1: 1, \mathrm{R}_{\mathrm{f}}=0.62\right)$ to afford $6 \mathrm{a}(0.61 \mathrm{~g}, 84.3 \%)$. Compound 6b-6d were prepared similarly.

6a: ${ }^{1} \mathrm{H}$ NMR $\delta 2.16-2.29(\mathrm{~m}, 1 \mathrm{H}), 3.34(\mathrm{~s}, 6 \mathrm{H}), 3.47(\mathrm{~d}$, $4 \mathrm{H}, J=6.2 \mathrm{~Hz}), 3.54(\mathrm{~d}, 2 \mathrm{H}, J=5.9 \mathrm{~Hz}), 4.53(\mathrm{~s}, 2 \mathrm{H}), 7.29-$ $7.38(\mathrm{~m}, 5 \mathrm{H}), \mathbf{6 b}$ : 'H NMR $\delta 1.17(\mathrm{t}, 6 \mathrm{H}, J=7.1 \mathrm{~Hz}) 2.04-$ $2.22(\mathrm{~m}, 1 \mathrm{H}), 3.40-3.55(\mathrm{~m}, 10 \mathrm{H}), 4.49(\mathrm{~s}, 2 \mathrm{H}), 7.27-7.34$ (m, 5H). 6c: 'H NMR $\delta 0.91(\mathrm{t}, 6 \mathrm{H}, J=7.1 \mathrm{~Hz}), 1.48-1.67$ (m, 4H), 2.19-2.28 (m, 1H), $3.37(\mathrm{t}, 4 \mathrm{H}, J=6.6 \mathrm{~Hz}), 3.40-$ $3.59(\mathrm{~m}, 6 \mathrm{H}), 4.52(\mathrm{~s}, 2 \mathrm{H}), 7.27-7.41(\mathrm{~m}, 5 \mathrm{H}) .6 \mathrm{~d}:{ }^{1} \mathrm{H}$ NMR $\delta 0.92(\mathrm{t}, 6 \mathrm{H}, J=7.3 \mathrm{~Hz}), 1.23-1.35(\mathrm{~m}, 4 \mathrm{H}), 1.38-1.54(\mathrm{~m}$, $4 \mathrm{H}$ ), $2.18-2.24$ (sept, $1 \mathrm{H}, J=5.9 \mathrm{~Hz}$ ), 3.41 (t, $4 \mathrm{H}, J=6.2$ $\mathrm{Hz}), 3.48(\mathrm{~d}, 4 \mathrm{H}, J=5.9 \mathrm{~Hz}), 3.54(\mathrm{~d}, 2 \mathrm{H}, J=5.9 \mathrm{~Hz}), 4.51$ $(\mathrm{s}, 2 \mathrm{H}), 7.26-7.38(\mathrm{~m}, 5 \mathrm{H})$.

Preparation of 3-alkoxy-2-(alkoxymethyl)-1-propanols 7. The synthesis of $7 \mathbf{a}$ is typical. To a methanol $(5 \mathrm{~mL})$ solution of $6 \mathrm{a}(0.60 \mathrm{~g}, 2.68 \mathrm{mmol}), 5 \% \mathrm{Pd}-\mathrm{C}$ catalyst $(0.10$ g) was added. The mixture was shaken at room temperature for $3 \mathrm{~h}$ under hydrogen atmosphere. The resulting mixture was filtered and washed with ether. The filtrate and washings were combined and concentrated. Chromatography on silica gel (hexanes/ether 1:1, $R_{i}=0.32$ ) gave 3-methoxy-2(methoxymethyl)-1-propanol (7a, $0.30 \mathrm{~g}, 82.6 \%$ ). Compound $7 \mathbf{b}-7 \mathbf{d}$ were prepared similarly.

7a: 'H NMR $\delta 2.06-2.16(\mathrm{~m}, 1 \mathrm{H}) 2.61(\mathrm{br} \mathrm{s}, 1 \mathrm{H}), 3.33$ (s, $6 \mathrm{H}), 3.47(\mathrm{~d}, 4 \mathrm{H}, J=6.2 \mathrm{~Hz}), 3.74(\mathrm{~d}, 2 \mathrm{H}, J=4.8 \mathrm{~Hz}) .7 \mathrm{~b}:$ 'H NMR $\delta 1.18(\mathrm{t}, 6 \mathrm{H}, J=7.1 \mathrm{~Hz}) 2.03-2.16(\mathrm{~m}, 1 \mathrm{H}) 2.54$ (br s, $1 \mathrm{H}), 3.42-3.55(\mathrm{~m}, 8 \mathrm{H}), 3.76(\mathrm{~d}, 2 \mathrm{H}, J=4.76) .7 \mathrm{c}:{ }^{1} \mathrm{H}$ NMR $\delta 0.89(\mathrm{t}, 6 \mathrm{H}, \mathrm{J}=7.3 \mathrm{~Hz}), 1.57(\mathrm{~m}, 4 \mathrm{H}), 2.06-2.12(\mathrm{~m}$, $1 \mathrm{H}), 2.56(\mathrm{br} \mathrm{s}, 1 \mathrm{H}), 3.37(\mathrm{t}, 4 \mathrm{H}, J=6.6 \mathrm{~Hz}), 3.52(\mathrm{~m}, 4 \mathrm{H})$, $3.75(\mathrm{~d}, 2 \mathrm{H}, J=4.8 \mathrm{~Hz}) .7 \mathrm{~d}$ : ${ }^{1} \mathrm{H}$ NMR $\delta 0.93(\mathrm{t}, 6 \mathrm{H}, J=7.0$ $\mathrm{Hz}), 1.28-1.39(\mathrm{~m}, 4 \mathrm{H}), 1.40-1.63(\mathrm{~m}, 4 \mathrm{H}), 2.06-2.17(\mathrm{~m}$, $1 \mathrm{H}), 2.52$ (br s, $1 \mathrm{H}), 3.44(\mathrm{t}, 4 \mathrm{H}, J=6.2 \mathrm{~Hz}), 3.54(\mathrm{~m}, 4 \mathrm{H})$, $3.78(\mathrm{~d}, 2 \mathrm{H}, J=5.1 \mathrm{~Hz})$.

Preparation of 3-alkoxy-2-(alkoxymethyl)-1-propyl 4benzyloxybenzoates 9 . The synthesis of $9 \mathrm{a}$ is typical. To a dichloromethane $(4 \mathrm{~mL})$ solution of $\mathrm{N}, \mathrm{N}$-dicyclohexylcarbodiimide (DCC, $0.32 \mathrm{~g}, 1.55 \mathrm{mmol}$ ) and 4-dimethylaminopyridine (DMAP, $0.10 \mathrm{~g}, 0.82 \mathrm{mmol}$ ) were added 4benzyloxybenzoic acid $(8,0.33 \mathrm{~g}, 1.44 \mathrm{mmol})$ and the alcohol $7 \mathbf{a}(0.18 \mathrm{~g}, 1.31 \mathrm{mmol})$. The reaction mixture was heated to reflux for 1 day. The precipitates were filtered and washed with dichloromethane. The filtrate was washed with aqueous $\mathrm{NaHCO}_{3}$ solution and then dried $\left(\mathrm{Na}_{2} \mathrm{SO}_{4}\right)$, concentrated. The residue was purified by column chromatography (silica gel, hexane/ether $\left.1: 1, R_{\mathrm{f}}=0.60\right)$ to afford $9 \mathrm{a}(0.33 \mathrm{~g}$, $71.2 \%$ ). Compound $9 \mathrm{~b}-9 \mathrm{~d}$ were prepared similarly.

9a: 'H NMR $\delta 2.35$ (sept, $1 \mathrm{H}, J=6.2 \mathrm{~Hz}), 3.37(\mathrm{~s}, 6 \mathrm{H}$ ), 3.51 (d, $4 \mathrm{H}, J=6.2 \mathrm{~Hz}$ ), 4.37 (d, $2 \mathrm{H}, J=6.2 \mathrm{~Hz}), 5.14$ (s, $2 \mathrm{H}), 7.02(\mathrm{~d}, 2 \mathrm{H}, J=8.8 \mathrm{~Hz}), 7.38-7.46(\mathrm{~m}, 4 \mathrm{H}), 8.01(\mathrm{~d}$, $2 \mathrm{H}, J=8.8 \mathrm{~Hz}) .9 \mathrm{~b}:{ }^{1} \mathrm{H}$ NMR $\delta 1.20(\mathrm{t}, 6 \mathrm{H}, J=7.0 \mathrm{~Hz}), 2.37$ (sept, $1 \mathrm{H}, J=5.9 \mathrm{~Hz}), 3.42-3.54(\mathrm{~m}, 8 \mathrm{H}), 4.39(\mathrm{~d}, 2 \mathrm{H}, J=$ $5.9 \mathrm{~Hz}), 5.14(\mathrm{~s}, 2 \mathrm{H}), 7.02(\mathrm{~d}, 2 \mathrm{H}, J=8.8 \mathrm{~Hz}), 7.38-7.46(\mathrm{~m}$, $5 \mathrm{H}), 8.01(\mathrm{~d}, 2 \mathrm{H}, J=8.8 \mathrm{~Hz}) .9 \mathrm{c}$ : 'H NMR $\delta 0.92(\mathrm{t}, 6 \mathrm{H}, J=$ $7.5 \mathrm{~Hz}$ ), $1.50-1.66(\mathrm{~m}, 4 \mathrm{H}), 2.36$ (sept, $1 \mathrm{H}, J=5.9 \mathrm{~Hz}$ ), 3.39 $(\mathrm{t}, 4 \mathrm{H}, J=6.6 \mathrm{~Hz}), 3.55(\mathrm{~d}, 4 \mathrm{H}, J=5.9 \mathrm{~Hz}), 4.39(\mathrm{~d}, 2 \mathrm{H}, J=$ $5.9 \mathrm{~Hz}), 5.14(\mathrm{~s}, 2 \mathrm{H}), 7.01(\mathrm{~d}, 2 \mathrm{H}, J=8.8 \mathrm{~Hz}), 7.38-7.47(\mathrm{~m}$, $5 \mathrm{H}), 8.00(\mathrm{~d}, 2 \mathrm{H}, J=8.8 \mathrm{~Hz}) .9 \mathrm{~d}$ : 'H NMR $\delta 0.94(\mathrm{t}, 6 \mathrm{H}, J=$ $7.3 \mathrm{~Hz}), 1.30-1.46(\mathrm{~m}, 4 \mathrm{H}), 1.51-1.65(\mathrm{~m}, 4 \mathrm{H}), 2.39 .4$ (sept, $1 \mathrm{H}, J=5.9 \mathrm{~Hz}), 3.45(\mathrm{t}, 4 \mathrm{H}, J=6.4 \mathrm{~Hz}), 3.56(\mathrm{~d}, 4 \mathrm{H}, J=5.9$ $\mathrm{Hz}), 4.40(\mathrm{~d}, 2 \mathrm{H}, J=5.9 \mathrm{~Hz}), 5.14(\mathrm{~s}, 2 \mathrm{H}), 7.02(\mathrm{~d}, 2 \mathrm{H}, J=$ $9.2 \mathrm{~Hz}), 7.35-7.48(\mathrm{~m}, 5 \mathrm{H}), 8.03(\mathrm{~d}, 2 \mathrm{H}, J=9.2 \mathrm{~Hz})$.

Preparation of 3-alkoxy-2-(alkoxymethyl)-1-propyl 4hydroxybenzontes 10. The synthesis of $10 \mathrm{a}$ is typical. To a solution of $9 \mathrm{a}(0.32 \mathrm{~g}, 0.93 \mathrm{mmol})$ in methanol $(5 \mathrm{~mL}) 5 \%$ Pd-C catalyst $(0.10 \mathrm{~g})$ was added. The mixture was shaken at room temperature for $3 \mathrm{~h}$ under hydrogen. The mixture was filtered and washed with ether. The filtrate and washings were concentrated and chromatographed on silica gel (hexanes/ether 1:1, $\mathrm{R}_{\mathrm{f}}=0.32$ ) to give 3-methoxy-2(methoxymethyl)-1-propyl 4-hydroxybenzoate (10a, $0.21 \mathrm{~g}$, $89.0 \%$ ). Compound 10b-10d were prepared similarly.

10a: 'H NMR $\delta 2.31-2.41(\mathrm{~m}, 1 \mathrm{H}), 3.35(\mathrm{~s}, 6 \mathrm{H}), 3.51(\mathrm{~d}$, $4 \mathrm{H}, J=5.9 \mathrm{~Hz}), 4.35(\mathrm{~d}, 2 \mathrm{H}, J=6.2 \mathrm{~Hz}), 6.84(\mathrm{~d}, 2 \mathrm{H}, J=8.8$ 
$\mathrm{Hz}), 7.91(\mathrm{~d}, 2 \mathrm{H}, J=8.8 \mathrm{~Hz}) .10 \mathrm{~b}:{ }^{1} \mathrm{H}$ NMR $\delta 1.20(\mathrm{t}, 6 \mathrm{H}, J$ $=7.0 \mathrm{~Hz}), 2.34-2.42(\mathrm{~m}, 1 \mathrm{H}), 3.47-3.59(\mathrm{~m}, 8 \mathrm{H}), 4.39(\mathrm{~d}$, $2 \mathrm{H}, J=5.9 \mathrm{~Hz}), 6.85(\mathrm{~d}, 2 \mathrm{H}, J=8.8 \mathrm{~Hz}), 7.91(\mathrm{~d}, 2 \mathrm{H}, J=8.8$ $\mathrm{Hz}) .10 \mathrm{c}:{ }^{1} \mathrm{H}$ NMR $\delta 0.92(\mathrm{t}, 6 \mathrm{H}, \mathrm{J}=7.5 \mathrm{~Hz}), 1.51-1.68(\mathrm{~m}$, $4 \mathrm{H}), 2.39$ (sept, $1 \mathrm{H}, J=5.9 \mathrm{~Hz}$ ), 3.41 (t, $4 \mathrm{H}, J=6.6 \mathrm{~Hz}$ ), $3.57(\mathrm{~d}, 4 \mathrm{H}, J=5.9 \mathrm{~Hz}), 4.38(\mathrm{~d}, 2 \mathrm{H}, J=5.9 \mathrm{~Hz}), 6.86(\mathrm{~d}$, $2 \mathrm{H}, J=8.8 \mathrm{~Hz}$ ), 7.92 (d, $2 \mathrm{H}, J=8.8 \mathrm{~Hz}) .10 \mathrm{~d}$ : 'H NMR $\delta$ $0.94(\mathrm{t}, 6 \mathrm{H}, J=7.3), 1.30-1.46(\mathrm{~m}, 4 \mathrm{H}), 1.51-1.65(\mathrm{~m}, 4 \mathrm{H})$, 2.39 (sept, $1 \mathrm{H}, J=5.9 \mathrm{~Hz}$ ), 3.45 (t, $4 \mathrm{H}, J=6.4 \mathrm{~Hz}$ ), 3.56 (d, $4 \mathrm{H}, J=5.9 \mathrm{~Hz}), 4.40(\mathrm{~d}, 2 \mathrm{H}, J=5.9 \mathrm{~Hz}), 6.86(\mathrm{~d}, 2 \mathrm{H}, J=9.2$ $\mathrm{Hz}), 7.91(\mathrm{~d}, 2 \mathrm{H}, J=9.2 \mathrm{~Hz})$.

Preparation of 3-alkoxy-2-(alkoxymethyl)-1-propyl 4(4'-alkyloxybiphenyl-4-carbonyloxy)benzoates 12 . The synthesis of 12a is typical. To a solution of 4-hydroxybenzoate 10a $(0.20 \mathrm{~g}, 0.79 \mathrm{mmol})$, DCC $(0.20 \mathrm{~g}, 0.97$ mmol), and DMAP (0.08 g, $0.65 \mathrm{mmol})$ was added 4-(4'octyloxyphenyl)benzoic acid (11a, $0.29 \mathrm{~g}, 0.88 \mathrm{mmol})$. The reaction mixture was refluxed for 1 day. The precipitate was filtered and washed with dichloromethane. The filtrate was washed with aqueous $\mathrm{NaHCO}_{3}$ solution, dried $\left(\mathrm{Na}_{2} \mathrm{SO}_{4}\right)$, and concentrated. The crude product was chromatographed on silica gel (hexanes/ether 1:1, $\left.\mathrm{R}_{\mathrm{f}}=0.53\right)$ to give 12a. $(0.35 \mathrm{~g}$, $78.5 \%$ ). Compound $12 \mathrm{~b}-12 \mathrm{~h}$ were prepared similarly.

12a (8CCMe): 'H NMR $\delta 0.91(\mathrm{t}, 3 \mathrm{H}, J=7.0 \mathrm{~Hz}), 1.31$ $1.59(\mathrm{~m}, 10 \mathrm{H}), 1.75-1.84(\mathrm{~m}, 2 \mathrm{H}), 2.40$ (sept, $1 \mathrm{H}, J=5.9$ $\mathrm{Hz}), 3.38(\mathrm{~s}, 6 \mathrm{H}), 3.53(\mathrm{~d}, 4 \mathrm{H}, J=5.9 \mathrm{~Hz}), 4.04(\mathrm{t}, 2 \mathrm{H}, J=$ $6.6 \mathrm{~Hz}), 4.43$ (d, $2 \mathrm{H}, J=5.9 \mathrm{~Hz}), 7.03(\mathrm{~d}, 2 \mathrm{H}, J=8.8 \mathrm{~Hz}$ ), 7.34 (d, $2 \mathrm{H}, J=8.8 \mathrm{~Hz}), 7.62(\mathrm{~d}, 2 \mathrm{H}, J=8.8 \mathrm{~Hz}), 7.72(\mathrm{~d}$, $2 \mathrm{H}, J=8.4 \mathrm{~Hz}), 8.15(\mathrm{~d}, 2 \mathrm{H}, J=8.8 \mathrm{~Hz}), 8.25(\mathrm{~d}, 2 \mathrm{H}, J=8.8$ $\mathrm{Hz}) ;{ }^{13} \mathrm{C}-\mathrm{NMR} \delta 165.4,164.3,159.3,154.4,146.0,131.6$, $131.0,130.6,128.2,127.7,126.8,126.5,121.7,114.8,70.8$, $68.2,63.5,59.1,39.6,32.0,29.5,29.4,26.2,22.9,14.3$; Anal. calc. for $\mathrm{C}_{34} \mathrm{H}_{42} \mathrm{O}_{7}: \mathrm{C} 72.57, \mathrm{H} 7.52$; found: $\mathrm{C} 72.51, \mathrm{H}$ 7.53 .

12b (9CCMe): 'H NMR $\delta 0.88(\mathrm{t}, 3 \mathrm{H}, J=6.6 \mathrm{~Hz}), 1.27-$ $1.57(\mathrm{~m}, 12 \mathrm{H}), 1.78-1.81(\mathrm{~m}, 2 \mathrm{H}), 2.35-2.41(\mathrm{~m}, 1 \mathrm{H}), 3.35$ $(\mathrm{s}, 6 \mathrm{H}), 3.51(\mathrm{~d}, 4 \mathrm{H}, J=6.2 \mathrm{~Hz}), 4.01(\mathrm{t}, 2 \mathrm{H}, J=6.6 \mathrm{~Hz}$ ), $4.40(\mathrm{~d}, 2 \mathrm{H}, J=5.9 \mathrm{~Hz}), 7.00(\mathrm{~d}, 2 \mathrm{H}, J=8.8 \mathrm{~Hz}), 7.32$ (d, $2 \mathrm{H}, J=8.8 \mathrm{~Hz}), 7.60(\mathrm{~d}, 2 \mathrm{H}, J=8.8 \mathrm{~Hz}), 7.70(\mathrm{~d}, 2 \mathrm{H}, J=8.8$ $\mathrm{Hz}), 8.12(\mathrm{~d}, 2 \mathrm{H}, J=8.8 \mathrm{~Hz}), 8.23(\mathrm{~d}, 2 \mathrm{H}, J=8.8 \mathrm{~Hz}) ;{ }^{13} \mathrm{C}-$ NMR $\delta 165.7,164.6,159.7,154.8,146.3,131.9,131.2$, $130.8,128.4,128.0,127.1,126.7,121.8,115.1,70.9,68.2$, $63.6,59.1,39.6,31.9,29.5,29.4,29.3,26.1,22.7,14.1$; Anal. calc. for $\mathrm{C}_{3,5} \mathrm{H}_{44} \mathrm{O}_{7}: \mathrm{C} 72.89, \mathrm{H} 7.69$; found: $\mathrm{C} 72.99, \mathrm{H}$ 7.77.

$12 \mathrm{c}(10 \mathrm{CCMe})$ : 'H NMR $\delta 0.90(\mathrm{t}, 3 \mathrm{H}, J=6.4 \mathrm{~Hz}), 1.30-$ $1.62(\mathrm{~m}, 14 \mathrm{H}), 1.76-1.87(\mathrm{~m}, 2 \mathrm{H}), 2.37-2.46(\mathrm{~m}, 1 \mathrm{H}), 3.38$ $(\mathrm{s}, 6 \mathrm{H}), 3.53(\mathrm{~d}, 4 \mathrm{H}, J=6.2 \mathrm{~Hz}), 4.04(\mathrm{t}, 2 \mathrm{H}, J=6.6 \mathrm{~Hz})$, $4.42(\mathrm{~d}, 2 \mathrm{H}, J=5.9 \mathrm{~Hz}), 7.02(\mathrm{~d}, 2 \mathrm{H}, J=8.8 \mathrm{~Hz}), 7.34$ (d, $2 \mathrm{H}, J=8.8 \mathrm{~Hz}), 7.62(\mathrm{~d}, 2 \mathrm{H}, J=8.8 \mathrm{~Hz}), 7.72(\mathrm{~d}, 2 \mathrm{H}, J=8.4$ $\mathrm{Hz}), 8.14(\mathrm{~d}, 2 \mathrm{H}, J=8.8 \mathrm{~Hz}), 8.25(\mathrm{~d}, 2 \mathrm{H}, J=8.4 \mathrm{~Hz}) ;{ }^{13} \mathrm{C}-$ NMR $\delta 165.4,164.3,159.3,154.4,146.0,131.6,131.0$, $130.6,128.2,127.7,126.7,126.5,121.7,114.8,70.8,68.2$, 63.5, 59.1, 39.6, 32.1, 29.7, 29.6, 29.5, 29.4, 26.2, 22.9, 14.4; Anal. calc. for $\mathrm{C}_{36} \mathrm{H}_{46} \mathrm{O}_{7}: \mathrm{C} 73.19, \mathrm{H} 7.85$; found: $\mathrm{C} 73.47 \mathrm{H}$ 7.95 .

12d (9CCEt): ${ }^{1} \mathrm{H}$ NMR $\delta 0.89$ (t, $\left.3 \mathrm{H}, J=7.0 \mathrm{~Hz}\right), 1.19$ (t, $6 \mathrm{H}, J=7.0 \mathrm{~Hz}), 1.12-1.51(\mathrm{~m}, 12 \mathrm{H}), 1.78-1.85(\mathrm{~m}, 2 \mathrm{H})$,
2.35-2.41 (m, 1H), 3.44-3.56 (m, 8H), $4.01(\mathrm{t}, 2 \mathrm{H}, J=6.6$ $\mathrm{Hz}), 4.42$ (d, $2 \mathrm{H}, J=5.9 \mathrm{~Hz}$ ), 7.00 (d, $2 \mathrm{H}, J=8.8 \mathrm{~Hz}$ ), 7.32 $(\mathrm{d}, 2 \mathrm{H}, J=8.8 \mathrm{~Hz}), 7.60(\mathrm{~d}, 2 \mathrm{H}, J=8.8 \mathrm{~Hz}), 7.70(\mathrm{~d}, 2 \mathrm{H}, J=$ $8.8 \mathrm{~Hz}), 8.12(\mathrm{~d}, 2 \mathrm{H}, J=8.8 \mathrm{~Hz}), 8.23(\mathrm{~d}, 2 \mathrm{H}, J=8.4 \mathrm{~Hz})$; ${ }^{13}$ C-NMR $\delta 165.8,159.7,154.7,146.3,131.9,131.2,130.8$, $129.1,128.4,128.0,127.1,126.7,121.8,115.1,68.7,68.2$, 66.6, 63.9, 39.7, 31.9, 29.6, 29.4, 29.3, 26.1, 22.7, 15.1, 14.1; Anal. calc. for $\mathrm{C}_{37} \mathrm{H}_{48} \mathrm{O}_{7}: \mathrm{C} 73.48, \mathrm{H} 8.00$; found: $\mathrm{C} 73.23, \mathrm{H}$ 8.06 .

12e (10CCEt): ${ }^{1} \mathrm{H} N M R \quad \delta 0.90(\mathrm{t}, 3 \mathrm{H}, J=5.9 \mathrm{~Hz}), 1.21(\mathrm{t}$, $6 \mathrm{H}, J=6.4 \mathrm{~Hz}), 1.29-1.76(\mathrm{~m}, 14 \mathrm{H}), 1.84-1.89(\mathrm{~m}, 2 \mathrm{H})$, 2.37-2.46 (m, 1H), 3.46-3.72 (m, 8H), $4.03(\mathrm{t}, 2 \mathrm{H}, J=6.6$ $\mathrm{Hz}), 4.44(\mathrm{~d}, 2 \mathrm{H}, J=5.9 \mathrm{~Hz}), 7.03(\mathrm{~d}, 2 \mathrm{H}, J=8.8 \mathrm{~Hz}), 7.34$ $(\mathrm{d}, 2 \mathrm{H}, J=8.8 \mathrm{~Hz}), 7.62(\mathrm{~d}, 2 \mathrm{H}, J=8.4 \mathrm{~Hz}), 7.72(\mathrm{~d}, 2 \mathrm{H}, J=$ $8.1 \mathrm{~Hz}), 8.15$ (d, $2 \mathrm{H}, J=8.4 \mathrm{~Hz}), 8.25$ (d, $2 \mathrm{H}, J=8.4 \mathrm{~Hz}$ ); ${ }^{13}$ C-NMR $\delta 165.8,164.6,159.7,154.7,146.3,131.9,131.2$, $130.8,128.4,128.1,127.1,126.7,121.8,115.1,68.7,68.2$, $66.6,63.9,39.7,31.9,29.5,29.4,29.3,29.2,26.1,22.7,15.1$, 14.1; Anal. calc. for $\mathrm{C}_{38} \mathrm{H}_{50} \mathrm{O}_{7}: \mathrm{C} 73.76, \mathrm{H} 8.14$; found: $\mathrm{C}$ $74.15, \mathrm{H} 8.28$.

$12 f(9 C C P r):$ 'H NMR $\delta 0.87-0.95(\mathrm{~m}, 9 \mathrm{H}), 1.28-1.66(\mathrm{~m}$, $16 \mathrm{H}), 1.81-1.84(\mathrm{~m}, 2 \mathrm{H}), 2.36-2.41(\mathrm{~m}, 1 \mathrm{H}), 3.39(\mathrm{t}, 4 \mathrm{H}, J=$ $6.6 \mathrm{~Hz}), 3.54(\mathrm{~d}, 4 \mathrm{H}, J=6.6 \mathrm{~Hz}), 4.01$ (t, $2 \mathrm{H}, J=6.6 \mathrm{~Hz}$ ), 4.42 (d, $2 \mathrm{H}, J=5.9 \mathrm{~Hz}), 7.00$ (d, $2 \mathrm{H}, J=8.8 \mathrm{~Hz}), 7.32$ (d, $2 \mathrm{H}, J=8.8 \mathrm{~Hz}), 7.65(\mathrm{~d}, 2 \mathrm{H}, J=8.8 \mathrm{~Hz}), 7.70(\mathrm{~d}, 2 \mathrm{H}, J=8.8$ $\mathrm{Hz}), 8.15(\mathrm{~d}, 2 \mathrm{H}, J=8.8 \mathrm{~Hz}), 8.23(\mathrm{~d}, 2 \mathrm{H}, J=8.8 \mathrm{~Hz}) ;{ }^{13} \mathrm{C}-$ NMR $\delta 165.4,164.3,159.3,154.4,146.0,131.6,131.0$, $130.6,128.2,127.8,126.8,126.5,121.6,114.8,73.0,68.8$, $68.2,63.9,39.8,32.0,29.7,29.6,29.4,26.2,23.0,22.9,14.3$, 10.8; Anal. calc. for $\mathrm{C}_{39} \mathrm{H}_{52} \mathrm{O}_{7}: \mathrm{C} 74.02, \mathrm{H} 8.28$; found: $\mathrm{C}$ $73.75, \mathrm{H} 8.42$.

12g (10CCPr): 'H NMR $\delta 0.87-0.97(\mathrm{~m}, 9 \mathrm{H}), 1.29-1.69$ $(\mathrm{m}, 18 \mathrm{H}), 1.80-1.87(\mathrm{~m}, 2 \mathrm{H}), 2.38-2.44(\mathrm{~m}, 1 \mathrm{H}), 3.41(\mathrm{t}, 4 \mathrm{H}$, $J=6.6 \mathrm{~Hz}), 3.56(\mathrm{~d}, 4 \mathrm{H}, J=6.2 \mathrm{~Hz}), 4.03(\mathrm{t}, 2 \mathrm{H}, J=6.4 \mathrm{~Hz})$, $4.44(\mathrm{~d}, 2 \mathrm{H}, J=5.9 \mathrm{~Hz}), 7.02(\mathrm{~d}, 2 \mathrm{H}, J=8.8 \mathrm{~Hz}), 7.34(\mathrm{~d}$, $2 \mathrm{H}, J=8.8 \mathrm{~Hz}), 7.62(\mathrm{~d}, 2 \mathrm{H}, J=8.4 \mathrm{~Hz}), 7.72(\mathrm{~d}, 2 \mathrm{H}, J=8.4$ $\mathrm{Hz}), 8.15(\mathrm{~d}, 2 \mathrm{H}, J=8.4 \mathrm{~Hz}), 8.25(\mathrm{~d}, 2 \mathrm{H}, J=8.4 \mathrm{~Hz}) ;{ }^{13} \mathrm{C}-$ NMR $\delta 165.4,164.3,159.3,154.3,146.0,131.6,130.9$, $130.6,128.2,127.8,126.7,126.4,121.6,114.8,73.0,68.8$, $68.2,63.8,39.7,32.0,29.7,29.54,29.47,29.4,26.2,23.0$, $22.9,14.3,10.8$; Anal. calc. for $\mathrm{C}_{40} \mathrm{H}_{54} \mathrm{O}_{7}: \mathrm{C} 74.27, \mathrm{H} 8.41$; found: C 74.29, H 8.54 .

12h (10CCBu): ${ }^{1} \mathrm{H}$ NMR $\delta 0.87-0.96(\mathrm{~m}, 9 \mathrm{H}), 1.30-1.63$ $(\mathrm{m}, 22 \mathrm{H}), 1.80-1.87(\mathrm{~m}, 2 \mathrm{H}), 2.37-2.43(\mathrm{~m}, 1 \mathrm{H}), 3.45(\mathrm{t}, 4 \mathrm{H}$, $J=6.6 \mathrm{~Hz}), 3.56(\mathrm{~d}, 4 \mathrm{H}, J=6.6 \mathrm{~Hz}), 4.04(\mathrm{t}, 2 \mathrm{H}, J=6.6 \mathrm{~Hz})$, $4.44(\mathrm{~d}, 2 \mathrm{H}, J=5.9 \mathrm{~Hz}), 7.03(\mathrm{~d}, 2 \mathrm{H}, J=8.8 \mathrm{~Hz}), 7.34(\mathrm{~d}$, $2 \mathrm{H}, J=8.8 \mathrm{~Hz}), 7.62(\mathrm{~d}, 2 \mathrm{H}, J=8.8 \mathrm{~Hz}), 7.72(\mathrm{~d}, 2 \mathrm{H}, J=8.8$ $\mathrm{Hz}), 8.15(\mathrm{~d}, 2 \mathrm{H}, J=8.8 \mathrm{~Hz}), 8.25(\mathrm{~d}, 2 \mathrm{H}, J=8.8 \mathrm{~Hz}) ;{ }^{13} \mathrm{C}$ NMR $\delta 165.4,164.3,159.3,154.3,146.0,131.6,130.9$, $130.6,128.2,127.8,126.8,126.4,121.6,114.8,71.1,68.8$, $68.2,63.8,39.7,32.0,31.9,29.7,29.55,29.47,29.4,26.2,1$, $22.9,19.5,14.3,14.1$; Anal. calc. for $\mathrm{C}_{42} \mathrm{H}_{58} \mathrm{O}_{7}: \mathrm{C} 74.74, \mathrm{H}$ 8.66; found: C 74.36, $\mathrm{H} 8.74$.

\section{Results and Discussion}

Synthesis of new achiral swallow-tailed liquid crystals 


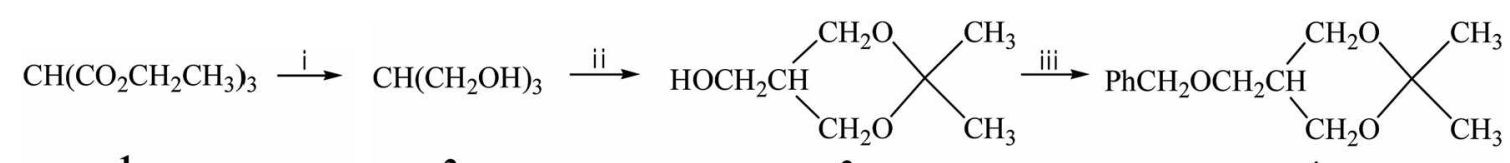<smiles>C#CCOCC(CO)CO</smiles>

5

12



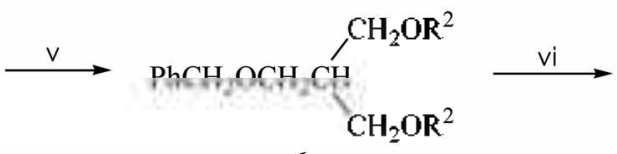

6<smiles>[R]OCC(CO)CO[R]</smiles>

4

Scheme 1. i. $\mathrm{H}_{3} \mathrm{BS}\left(\mathrm{Mc}_{2}\right)$, ii. $\left(\mathrm{CH}_{3} \mathrm{O}\right)_{2} \mathrm{C}\left(\mathrm{CH}_{3}\right)_{2} \mathrm{TsOH}$, iii. $\mathrm{NaH} / \mathrm{THF}$ then $\mathrm{PhCH} 2 \mathrm{Br}$, iv. H, H-O, v. NaH (2.5 equiv)/DMF then R-Br (3-5 equiv), vi. $\mathrm{H}-/ \mathrm{Pd}-\mathrm{C}$, vii. $\mathrm{PhCH}_{2} \mathrm{O}-\mathrm{COOH}(\mathbf{8}) / \mathrm{DCC}, \mathrm{DMAP}$, viii. $\mathrm{H}_{2} / \mathrm{Pd}-\mathrm{C}$, ix. $\mathrm{R}^{\prime} \mathrm{C}-\mathrm{COOH}(\mathbf{l 1})$, DCC, DMAP

derived from 3-alkoxy-2-(alkoxymethyl)-1-propanol where alkyl is methyl, ethyl, propyl, and butyl was conducted by utilizing known reactions. 3-Alkoxy-2-(alkoxymethyl)-Ipropanols 7 were prepared from triethylmethanetricarboxylate (1) via a six step reaction sequence (Scheme 1). The alcohols 7 were esterified with 4-benzyloxybenzoic acid 8 in the presence of 1,3-dicyclohexyl carbodiumide $(\mathrm{DCC})$ and N,N-dimethylaminopyridine (DMAP).

4-Benzyloxybenzoates 9 were debenzylated by Pdcatalyzed hydrogenation reaction. Esterification of the resulting 4-hydroxybenzoates 10 with 4-alkyloxybiphenyl4-carboxylic acid 11 in the presence of DCC and DMAP afforded the final swallow-tailed liquid crystals in good yields. In the abbreviation of the final products $n C C R, n$ is the carbon number of the terminal alkoxy group $\left(R^{\prime}\right)$ at the biphenyl ring side and $\mathrm{R}$ is the alkyl group $\left(\mathrm{R}^{2}\right)$ in swallowtail of 12. The structures of the firlal products and intermediates were jdentified by ${ }^{1} \mathrm{H} N \mathrm{NM},{ }^{1 / 3} \mathrm{C} \mathrm{NMR}$, and elemental analysis.

The mesophase transition temperatures and enthalpies of the final compounds were determined by differential scanning calorimetry (DSC) in conjunction with optical polarizing microscopy. Mesophases were identified by observing the microscopic textures of the materials layered between two untreated glass plates. The results are summarized in Table 1.

The liquid crystals 12 were found to exhibit a wide temperature range for the 'antiferroelectric like' SmCalt phase with the phase sequence (I-SmA-SmCalt-Cr). The SmA phase displayed a batonnet texture. Further cooling of the smectic A phase of these compounds resulted in the formation of a schlieren texture characterized by the appearance of both two $(\mathrm{S}= \pm 1 / 2)$ and four-brush singularities ( $\mathrm{S}=$ \pm 1 ) as shown in Figure 1. The appearance of two brush singularities in the schlieren texture was reported to be diagnostic for the antiferroelectric-like phase. ${ }^{1}$ Compound 12e (10CCEt) did not exhibit a striated focal-conic or schlieren texture of the SmCalt phase. It showed an enantiotropic phase sequences of I-SmA-SmC-Cr. Among the liquid crystals, 12a (8CCMe), 12f ( 9 CCPr), and $12 \mathrm{~g}$ (10CCPr) were found to exhibit an unidentified SmCX phase between SmCalt and crystal phase. The SmCX phases appeared in the DSC thermograms, however they were not observed by the thermal optical polarized light microscopy.

In case $\mathrm{R}^{1}$ of $\mathbf{1 2}$ is nonyl, the clearing point, the SmASmCalt transition temperature, and melting point become lower as the $\mathrm{R}^{2}$ group of the swallow tail is elongated from methyl (12b), to ethyl (12d) and to propyl (12f). In case $\mathrm{R}^{1}$ of 12 is decyl, the same trend was observed. The similar trends were also observed in the achiral swallow-tailed liquid crystals derived from 1,3-dialkoxy-2-propanol. ${ }^{3}$ However, though the length of swallow-tailed chain increases the thermal stabilities of SmA and SmCalt are not largely changed with the elongation of swallow-tailed chair.

In order to investigate the potential use of the obtained bisalkoxy swallow-tailed compounds as a host in the antiferroelectric mixtures, a miscibility study was performed. The binary mixture of the swallow-tailed compound $12 \mathrm{~g}$ $(10 C \mathrm{CPr})$ and the chiral antiferroelectric liquid crysta] [(S)$\mathrm{MHPOBC}$ (A) were prepared by weighing each component 
Table 1. Phase transition temperatures $\left({ }^{\circ} \mathrm{C}\right)$ and enthalpies $\left(\Delta H / \mathrm{kJ}_{\mathrm{Inol}}{ }^{-1}\right)($ in italics) for the 3-alkoxy-2-(alkoxymethyl)-1-propyl $4-(4-$ alkoxybiphenyl-4-carbonyloxy)benzoates 12 on cooling

\begin{tabular}{|c|c|c|c|c|c|c|c|c|c|c|c|c|}
\hline Compound & $\mathrm{mp}$ & $\mathrm{Cr}$ & & $\operatorname{SinCX}$ & & SinCalt & & $\operatorname{SinC}$ & & $\operatorname{SmA}$ & & I \\
\hline 12a (8CCMe) & 85.3 & - & $\begin{array}{l}36.9 \\
7.35\end{array}$ & • & $\begin{array}{l}56.8 \\
0.26\end{array}$ & - & $\begin{array}{c}118.0 \\
0.10\end{array}$ & - & & - & $\begin{array}{c}148.3 \\
4.01\end{array}$ & - \\
\hline $12 b(9 \mathrm{CCMe})$ & 86.2 & - & $\begin{array}{l}59.1 \\
17.4\end{array}$ & - & & - & $\begin{array}{c}115.0 \\
0.10\end{array}$ & - & & • & $\begin{array}{c}141.4 \\
3.57\end{array}$ & - \\
\hline $12 \mathrm{c}(10 \mathrm{CCMe})$ & 79.1 & - & $\begin{array}{l}38.0 \\
12.4\end{array}$ & - & & - & $\begin{array}{c}113.8 \\
0.23\end{array}$ & - & & • & $\begin{array}{c}138.3 \\
4.10\end{array}$ & - \\
\hline 12d (9CCEt) & 72.9 & - & $\begin{array}{l}45.1 \\
3.29\end{array}$ & - & & - & $\begin{array}{c}104.7 \\
0.07\end{array}$ & - & & - & $\begin{array}{c}127.8 \\
3.81\end{array}$ & - \\
\hline $12 \mathrm{e}(10 \mathrm{CCEt})$ & 56.2 & - & $\begin{array}{l}11.4 \\
7.41\end{array}$ & - & & - & & • & $\begin{array}{l}99.2 \\
0.10\end{array}$ & • & $\begin{array}{c}121.3 \\
5.03\end{array}$ & - \\
\hline $12 \mathrm{f}(9 \mathrm{CCPr})$ & 71.3 & - & $\begin{array}{l}46.6 \\
18.2\end{array}$ & - & $\begin{array}{l}58.8 \\
0.04\end{array}$ & - & $\begin{array}{l}96.1 \\
0.13\end{array}$ & - & & • & $\begin{array}{c}117.0 \\
3.68\end{array}$ & - \\
\hline $12 \mathrm{~g}(10 C \mathrm{CP})$ & 46.1 & - & $\begin{array}{l}3.8 \\
12.0\end{array}$ & • & $\begin{array}{l}58.7 \\
0.05\end{array}$ & - & $\begin{array}{l}92.9 \\
0.29\end{array}$ & - & & - & $\begin{array}{c}111.5 \\
3.16\end{array}$ & - \\
\hline $12 \mathrm{~h}(10 \mathrm{CCBu})$ & 45.6 & - & $\begin{array}{c}8.6 \\
13.7\end{array}$ & - & & - & $\begin{array}{l}89.6 \\
0.22\end{array}$ & - & & - & $\begin{array}{c}109.0 \\
3.10\end{array}$ & - \\
\hline
\end{tabular}

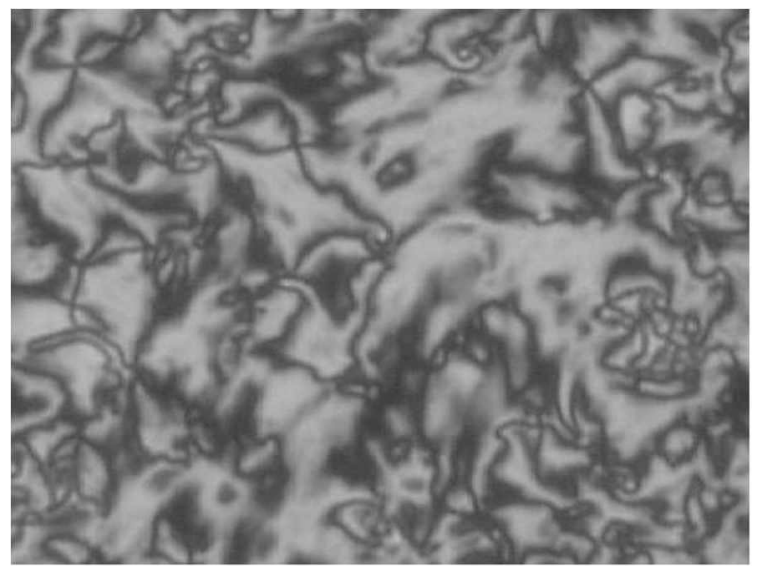

Figure 1. Schlieren texture of the SunCalt phase observed in $12 \mathrm{~g}$ (10CCPr) at $75.0^{\circ} \mathrm{C}$ on cooling exhibits both two-and four-brush singularities. into a clean glass vial, and dissolved with anhydrous dichloromethane. The dichloromethane was then evaporated and the last trace of solvent was under vacuum.

The DSC data for the bisalkoxy swallow-tailed compound $12 \mathrm{~g}$ (10CCPr), the branched alkyl chain swallow-tailed material (B), and the binary mixtures of each of them with the chiral antiferroeletric liquid crystal [(S)-MHPOBC, A] were summarized in Table 2 . The binary mixture of the chiral (S)-MHPOBC (A) with the bisalkoxy swallow-tailed liquid crystal $(\mathbf{1 2} \mathrm{g})$ showed a phase sequence of I-SmA$\mathrm{SmC}_{\mathrm{A}}-\mathrm{SmCX}-\mathrm{Cr}$. Its temperature range of $\mathrm{SmC}_{\mathrm{A}}$ was much broader than that of the binary mixture of the compound containing a branched alkyl swallow-tail (B) and the chiral liquid crystal $\mathbf{A} .^{1 d}$ In this particular case the smectic A phase exhibited a focal conic texture and the $\mathrm{SmC}_{\mathrm{A}}{ }^{*}$ phase were characterized by the schlieren texture with two- and four brush singularities shown in Figure 2.

Table 2. The transition temperatures and associated enthalpies ( $\triangle H / \mathrm{Jinol}^{-1}$ )(in italics) for the binary mixtures of $\mathbf{A}$ with $12 \mathrm{~g}$ ( IOCCPr) and A with $\mathbf{B}$

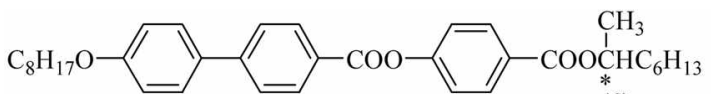

(S)

A $(S)$-MHPOBC

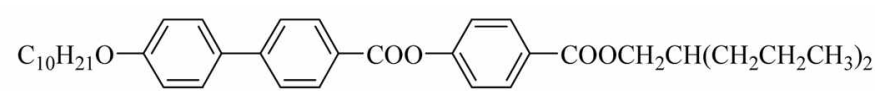

B

\begin{tabular}{|c|c|c|c|c|c|c|c|c|c|c|c|}
\hline Code & $\mathrm{Cr}$ & & SinCx & & SinCalt $/ \mathrm{SmC}_{\Lambda}{ }^{*}$ & & $\mathrm{SmC} / \mathrm{SmC}^{*}$ & & $\sin A$ & & I \\
\hline $12 \mathrm{~g}(10 \mathrm{CCPr})$ & - & $\begin{array}{c}3.8 \\
12.0\end{array}$ & - & $\begin{array}{l}58.7 \\
0.05\end{array}$ & - & $\begin{array}{l}92.9 \\
0.29\end{array}$ & - & & - & $\begin{array}{l}111.5 \\
3.16\end{array}$ & - \\
\hline B & - & $\begin{array}{l}60.4 \\
68.3\end{array}$ & - & & • & $\begin{array}{c}116.2 \\
1.4\end{array}$ & - & & • & $\begin{array}{c}136.9 \\
9.9\end{array}$ & - \\
\hline 12g $85 \% / \mathrm{A} 15 \%$ & - & $\begin{array}{c}-1.8 \\
3.2\end{array}$ & - & $\begin{array}{l}26.5 \\
0.06\end{array}$ & - & $\begin{array}{l}97.7 \\
0.1 I\end{array}$ & - & & - & $\begin{array}{c}122.5 \\
3.6\end{array}$ & - \\
\hline B $85 \% / A 15 \%$ & - & $\begin{array}{l}49.9 \\
52.4\end{array}$ & - & & • & $\begin{array}{c}119.2 \\
1.8\end{array}$ & - & & - & $\begin{array}{c}140.3 \\
12.5\end{array}$ & - \\
\hline $\mathbf{A}$ & - & 73.5 & - & & - & 121.0 & - & 123.0 & - & 151.5 & - \\
\hline
\end{tabular}




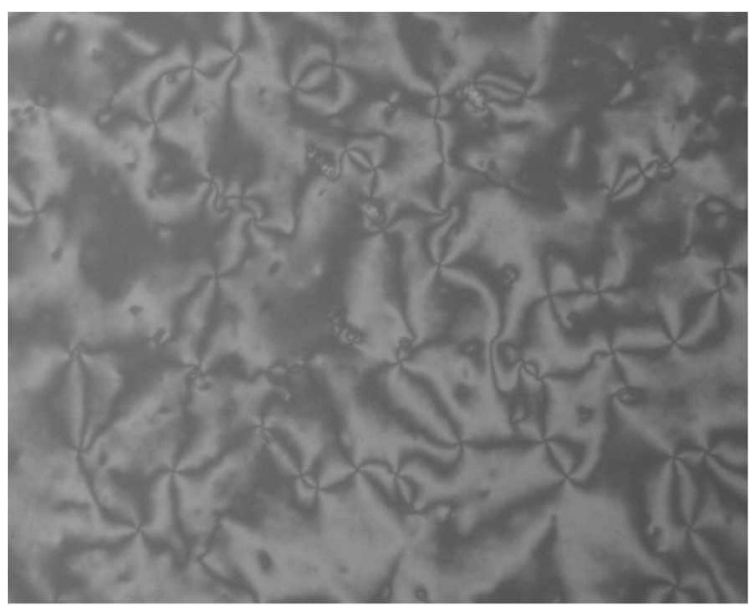

Figure 2. The schlieren texture of the $\mathrm{SInC}_{A}{ }^{*}$ phase observed in the mixture $12 \mathrm{~g} 85 / \mathrm{A} / 5$ shows two- and four-brush singularities.

\section{Conclusion}

New achiral swallow-tailed liquid crystals derived from 3alkoxy-2-(alkoxymethy])-1-propanol were prepared in good yields. These liquid crystals exhibit antiferroelectric-like smectic $\mathrm{C}$ phase at temperature lower than and temperature range broader than does compound bearing the corresponding branched alkyl swallow-tails. Investigation of the binary mixtures with a chiral antiferroelectric liquid crystal indicates that new achiral liquid crystals with bisalkoxy swallow-tails can be more useful as host components of antiferroelectric mixture.

Acknowledgements. This work was performed as part of Advanced Backbone IT technology development project supported by Ministry of Information \& Communication in Republic of Korea.

\section{References}

I. (a) Nishiyama, I.; Goodby, J. W. J. Mater. Chem. 1992, 2, 1015. (b) Ouchi, Y.; Yoshioka, Y.; Ishii, H.; Seki, K.; Kitamura, M.; Noyori, R.; Takanishi, Y.; Nishiyama, I. J. Mater. Chem. 1995, 5 , 2297. (c) Booth, C. J.; Durmur, D. A.; Goodby, J. W.; Halely, J.; Toyne, K. J. Liq. Cryst, 1996, 20, 387, (d) Wu, S.-L.; Chiang, C.T, Liq. Civst, 2002, 29, 39, (e) Wu, S.-L.; Chen, Y.-P. Liq. Const. $2004,31,607$

2. (a) Wu, S.-L.; Hsieh, W.-J. Chem. Mater. 1999, M, 852. (b) Wu, S.-L.; Lin, C.-Y. Liq. Cnst. 2003, 30, 205. (c) Wu, S.-L.; Lin, C.Y. Liq. Cryst. 2003, 30, 471. (d) Wu, S.-L.; Chen, F.-D. Liq. Cryst. 2003, 30,991. (e) Wu, S.-L.; Lin, T.-C. Liq. Cinst. 2004, 31, 1469. (I) Wu, S.-L.; Lu, F.-C. Liq. Cnst. 2004, 3I, 1517. (g) Wu, S.-L.; Chen, R.-B. Liq. Cryst. 2004, 31, 1613. (h) Wu, S.-L.; Chung, J.Y. Liq. Crost. 2005, 32, 1. (i) Wu. S.-L.; Lin, C.-Y. Liq. Crst. 2005, 32, 749. (j) Wu, S.-L.; Chen. K.-T. Liq. Cnst. 2006, 33, 573.

3. Kang, K.-T.; Lee, S. K.; Park, C. W.; Cho, S. H.; Lee, J. G.; Choi, S.-K.; Kim, Y, B. Bull, Korean Chem. Soc, 2006, 27, 1364.

4. Harnden, M. R.; Wyall, P. G; Boyd, M. R.; Suton, D. J. Med. Chem. $1990,33,187$. 